American Journal of Applied Mathematics
2020; 8(1): $29-33$
http://www.sciencepublishinggroup.com/j/ajam
doi: 10.11648 /j.ajam.20200801.15
ISSN: $2330-0043$ (Print); ISSN: $2330-006 \mathrm{X}$ (Online)

\title{
From the Continuity Problem of Set Potential to the Research of Male Gene Fragment
}

\author{
Zhu Rongrong \\ DIEG Mathematics Research of HR, Fudan University, Shanghai, China
}

\author{
Email address: \\ rongrongzhu1969@163.com
}

\section{To cite this article:}

Zhu Rongrong. From the Continuity Problem of Set Potential to the Research of Male Gene Fragment. American Journal of Applied Mathematics. Special Issue: Molecular Cellular Information Mathematics-Differential Incremental Equilibrium Geometry.

Vol. 8, No. 1, 2020, pp. 29-33. doi: 10.11648/j.ajam.20200801.15

Received: July 15, 2019; Accepted: October 8, 2019; Published: February 4, 2020

\begin{abstract}
The four mixed potentials belong to the category of hyperfinite theory and are discontinuous set potentials. From the basic frame structure of gene to the most excellent gene fragment of human male, while the basic frame structure of gene of female conforms to the basic frame rule of nature, male is only the supporting role; female is superior to male in the basic frame structure of gene; but male has the most excellent gene fragment of human. Therefore, it is important for human beings to establish the research center of male molecule (gene). The fragment gene has effects on memory, thinking and immunity, blood glucose, insulin and mental activity. However, the relationship between protein repair (function) and immunity enhancement is dependent on the function of memory gene and the angular velocity of thought dispersion, and the interaction between brain function and protein particle movement is formed. Protein repair embodies the core role of protein repair, which shows a chaotic order, and ensures the stability of every living tissue. Through the symmetry of group theory, this paper deeply analyzes the minimum limit kernel and its role, and there are countless homomorphic limit kernels in the minimum limit kernel, which can map and deduce the structure to build the hope of life when human life is greatly damaged, and can repair from the tiny place. The functional relationship between the movement of protein particles and cancer tissue will affect the life cycle, treatment measures and the change of impurities.
\end{abstract}

Keywords: Set Potential, Gene Basic Framework, Gene Fragment, Memory Gene,

Discrete Angular Velocity of Thought, Protein Repair, Minimum Limiting Nucleus, Cancer and Life Cycle

\section{Introduction}

\subsection{The Relationship Between the Basic Framework of Genes and the Minimum Framework of the Universe}

Whether the unity of continuity and local discontinuity exists. Secondly, whether there is a potential in the set of $N \rightarrow R$ numbers. Because the smallest element of the mixed potential is infinitesimal. So, there is always $a_{i}+$ $\lim \oint_{0^{-}}^{\infty} a_{i+\Delta \varepsilon}^{\uparrow \downarrow}$ with $a_{i}$ potential, because $a_{i}+\lim \oint_{0^{-}}^{\infty} a_{i+\Delta \varepsilon}^{\uparrow \downarrow}$ is a discontinuous potential [1].

$$
\left\{\begin{array}{l}
a_{i}+\lim \oint_{0^{-}}^{\infty} b_{i+\Delta \varepsilon} \rightarrow a_{i}+\lim \oint_{0^{+}}^{\infty} b_{i+\Delta \varepsilon} \\
a_{i}+\lim \oint_{0}^{\infty} b_{i+\Delta \varepsilon}^{\uparrow} \rightarrow a_{i}+\lim \oint_{0}^{\infty} b_{i+\Delta \varepsilon}^{\downarrow}
\end{array}\right.
$$

The four mixed potentials belong to the category of hyperfinite theory and are discontinuous set potentials [2]. And the basic frame structure of gene, male $X+\lim \oint_{0^{+}}^{\infty} \Delta y_{i+\varepsilon}^{-\infty}$ is the most excellent gene fragment of human. Women's basic frame structure of gene conforms to the basic frame rule of the whole universe (nature), while men are only supporting roles. Women are better than men in the basic framework of gene structure, but men's $X+\lim \oint_{0^{+}}^{\infty} y_{i+\Delta \varepsilon}^{-\infty}$ is the best gene fragment of human beings [3], so it is important for human beings to establish a "molecular (gene) Research Center for men".

$$
\left\{\begin{array}{l}
X+\lim \oint_{0^{-}}^{\infty} x y_{i+\Delta \varepsilon} \rightarrow X+\lim \oint_{0^{+}}^{\infty} x y_{i+\Delta \varepsilon} \\
X+\lim \oint_{0}^{\infty} x y_{i+\Delta \varepsilon}^{\uparrow} \rightarrow X+\lim \oint_{0}^{\infty} x y_{i+\Delta \varepsilon}^{\downarrow}
\end{array}\right.
$$

\subsection{The Effect of Fragment Gene on Memory, Thinking, Immunity, Blood Sugar, Insulin, Mental Activity and so on}

The combination of protein chains in human organs was analyzed by 


$$
\psi\left(\forall \nabla y_{\varepsilon}, \sum_{\varepsilon=1}^{n} \nabla y_{\varepsilon}^{\partial}\right) \rightarrow \prod_{j=1}^{m} \prod_{i=1}^{n} \oint\left(\forall G_{i} \leftrightarrow \forall N_{\varepsilon}\right)
$$

from which

$$
\operatorname{Cos}^{n-1}\left(\varphi_{1} \leftrightarrow P_{1}\right) \cdot \operatorname{Cos}^{n-2}\left(\varphi_{2} \leftrightarrow P_{2}\right) \cdot \operatorname{Cos}^{n-3}\left(\varphi_{3} \leftrightarrow P_{3}\right) \cdot \ldots \cdot \operatorname{Cos}^{1}\left(\varphi_{n} \leftrightarrow P_{n}\right)
$$

was separated as a trigonometric series (function) expansion, which conforms to the movement law of protein particles [4].

$D N A$ double stranded mathematical model, by extracting the nucleus,

$$
+2^{n} \cdot \sqrt{2}\left[\operatorname{Sin}\left(G_{i-1} \leftrightarrow N_{i-1}+\frac{\pi}{4}\right)\right] \quad \text { left } \text { chain, right }
$$

$$
\prod_{i=1}^{n} \operatorname{Cos}^{i-1}\left(\overline{\uparrow \downarrow}_{j=1}^{m} \varphi_{j} \leftrightarrow P_{j}\right) \rightarrow\left[\operatorname{Memory}\left(\psi_{i} \leftrightarrow \xi_{i}\right), H\left(L_{\alpha_{i}}^{\operatorname{Max}}, \omega_{i}^{\operatorname{Max}}\right)\right]
$$

Human fragment genes are related to immunity, blood glucose, insulin and mental activity. The relationship between protein repair (function) and immunity rise.

$$
\operatorname{Sin}\left(A_{\downarrow}^{\uparrow} \wedge E^{\uparrow} \leftrightarrow G^{\downarrow}+\frac{\pi}{4}\right) \leq \operatorname{Sin}\left[\left(A_{1}^{\uparrow}+\sum_{i=2}^{m} A_{2 i-1}^{! \downarrow} \leftrightarrow A_{2 i+1}^{! \uparrow}\right) \otimes\left[\sum A_{1}^{\uparrow}-\sum_{i=2}^{m} E_{i}^{\uparrow} \leftrightarrow G_{i}^{\downarrow}+\sum_{i=2}^{m} A_{2 i-1}^{! \downarrow} \leftrightarrow A_{2 i+1}^{! \uparrow} \otimes \sum_{i=2}^{m} E_{i}^{\uparrow} \leftrightarrow G_{i}^{\uparrow}\right]+\frac{\pi}{4}\right]
$$

Local initial value of immune system. Local initial value problems of immune and protein repair [8].

The relationship between protein repair (function) and weakened immunity.

$$
\operatorname{Sin}\left(A_{\downarrow}^{\uparrow} \wedge E^{\uparrow} \leftrightarrow G^{\downarrow}+\frac{\pi}{4}\right) \leq \operatorname{Sin}\left[\left(A_{1}^{\uparrow}+\sum_{i=2}^{m} A_{2 i-1}^{! \uparrow} \leftrightarrow A_{2 i+1}^{! \downarrow}\right) \otimes\left[\sum A_{1}^{\uparrow}-\sum_{i=2}^{m} E_{i}^{\uparrow} \leftrightarrow G_{i}^{\downarrow}+\sum_{i=2}^{m} A_{2 i-1}^{! \uparrow} \leftrightarrow A_{2 i+1}^{! \downarrow} \otimes \sum_{i=2}^{m} E_{i}^{\downarrow} \leftrightarrow G_{i}^{\downarrow}\right]+\frac{\pi}{4}\right]
$$

Local initial value of immune system. Local initial value problems of immune and protein repair [9].

Each index of protein repair and life [nucleus] embodies its core role in protein repair. It shows an order in chaos and guarantees the stability of all the organizations of every life [10].

$\operatorname{Sin}\left[\sum_{i=1, \varepsilon=1}^{m}\left(C_{i}, B_{i}\right)_{\downarrow}^{\uparrow} \wedge\left(E_{\varepsilon}^{\uparrow} \leftrightarrow G_{\varepsilon}^{\downarrow}\right)+i \cdot \frac{\pi}{4}\right] \leq$

$$
\begin{gathered}
{\left[\operatorname{Sin}\left[\frac{C_{1 \downarrow}^{\uparrow}}{2}+\sum_{i=2}^{m} C_{2 i-1}^{! \downarrow} \leftrightarrow C_{2 i+1}^{! \uparrow}\right] \operatorname{Cos}\left[\left(\sum_{i=2}^{m} C_{2 i-1}^{! \downarrow} \leftrightarrow C_{2 i+1}^{! \uparrow} \otimes \sum_{i=2}^{m} E_{i}^{\uparrow} \leftrightarrow G_{i}^{\downarrow}\right)+\sum_{i=2}^{m} \frac{\left(i C_{i}\right)_{2 i-1}^{\uparrow} \leftrightarrow\left(i C_{i}\right)_{2 i+1}^{\downarrow}}{2} \otimes \sum_{i=2}^{m} \frac{\left(i E_{i}\right)^{\uparrow} \leftrightarrow\left(i G_{i}\right)^{\downarrow}}{2}\right]\right]-} \\
{\left[\operatorname{Sin}\left[\frac{B_{1 \downarrow}^{\uparrow}}{2}+\sum_{i=2}^{m} B_{2 i-1}^{! \downarrow} \leftrightarrow B_{2 i+1}^{! \uparrow}\right] \operatorname{Cos}\left[\left(\sum_{i=2}^{m} B_{2 i-1}^{! \downarrow} \leftrightarrow B_{2 i+1}^{! \uparrow} \otimes \sum_{i=2}^{m} E_{i}^{\uparrow} \leftrightarrow G_{i}^{\downarrow}\right)+\sum_{i=2}^{m} \frac{\left(i B_{i}\right)_{2 i-1}^{\uparrow} \leftrightarrow\left(i B_{i}\right)_{2 i+1}^{\downarrow}}{2} \otimes \sum_{i=2}^{m} \frac{\left(i E_{i}\right)^{\uparrow} \leftrightarrow\left(i G_{i}\right)^{\downarrow}}{2}\right]\right](6)}
\end{gathered}
$$

The core is $\sum_{i=2}^{m} \frac{C_{2 i-1}^{! \uparrow} \leftrightarrow C_{2 i+1}^{! \downarrow}}{2} \otimes \sum_{i=2}^{m} \frac{E_{i}^{\uparrow} \leftrightarrow G_{i}^{\downarrow}}{2}, \sum_{i=2}^{m} \frac{B_{2 i-1}^{! \uparrow} \leftrightarrow B_{2 i+1}^{! \downarrow}}{2} \otimes \sum_{i=2}^{m} \frac{E_{i}^{\uparrow} \leftrightarrow G_{i}^{\downarrow}}{2}$

Through the symmetry of group theory [11], the minimum limit kernel is formed [12].

$$
\left\{\begin{aligned}
G_{2 i}^{\downarrow}\left(C-B^{\uparrow}\right) & =\frac{\left(i \cdot G_{i}\right)^{\downarrow}}{2} \otimes \frac{\left(i \cdot C_{i}\right)_{2 i}^{\uparrow} \wedge\left(i \cdot B_{i}\right)_{2 i}^{\uparrow}}{2} \\
G_{2 i}^{\uparrow}\left(C-B^{\downarrow}\right) & =\frac{\left(i \cdot G_{i}\right)^{\uparrow}}{2} \otimes \frac{\left(i \cdot C_{i}\right)_{2 i}^{\downarrow} \wedge\left(i \cdot B_{i}\right)_{2 i}^{\downarrow}}{2} \\
G_{2 i+1}^{\downarrow}\left(C-B^{\uparrow}\right) & =\frac{\left(i \cdot G_{i}\right)^{\downarrow}}{2} \otimes \frac{\left(i \cdot C_{i}\right)_{2 i+1}^{\uparrow} \wedge\left(i \cdot B_{i}\right)_{2 i+1}^{\uparrow}}{2} \\
G_{2 i+1}^{\uparrow}\left(C-B^{\downarrow}\right) & =\frac{\left(i \cdot G_{i}\right)^{\uparrow}}{2} \otimes \frac{\left(i \cdot C_{i}\right)_{2 i+1}^{\downarrow} \wedge\left(i \cdot B_{i}\right)_{2 i+1}^{\downarrow}}{2}
\end{aligned}\right.
$$

Minimum structure group nucleus protein fragment minimum gene frame unit [13].

$$
\left[\begin{array}{ll}
G_{2 i}^{\downarrow}\left(C-B^{\uparrow}\right) \leftrightarrow E_{2 i}^{\downarrow}\left(C-B^{\uparrow}\right) & G_{2 i+1}^{\downarrow}\left(C-B^{\uparrow}\right) \leftrightarrow E_{2 i+1}^{\downarrow}\left(C-B^{\uparrow}\right) \\
G_{2 i}^{\uparrow}\left(C-B^{\downarrow}\right) \leftrightarrow E_{2 i}^{\uparrow}\left(C-B^{\downarrow}\right) & G_{2 i+1}^{\uparrow}\left(C-B^{\downarrow}\right) \leftrightarrow E_{2 i+1}^{\uparrow}\left(C-B^{\downarrow}\right)
\end{array}\right]
$$

There are countless homomorphic limit nuclei in the minimum limit nucleus [14], which can obtain the hope of human life from the tiny places when human life is greatly damaged. The infinite arrangement of the minimum limit nuclei [15], the formation of repair proteins in life activities, and the regeneration in the metabolic process. 


$$
\psi\left(f_{\Sigma}\left(G_{2 i}^{\downarrow \uparrow}\right), f_{\Sigma}\left(E_{2 i}^{\downarrow \uparrow}\right)\right)=\sum\left(\frac{\left(i \cdot G_{i}\right)_{\uparrow}^{\downarrow}}{2} \otimes \frac{\left(i \cdot C_{i}\right)_{2 i-1}^{\uparrow \downarrow} \wedge\left(i \cdot B_{i}\right)_{2 i-1}^{\uparrow \downarrow}}{2} \leftrightarrow \frac{\left(i \cdot E_{i}\right)_{\uparrow}^{\downarrow}}{2} \otimes \frac{\left(i \cdot C_{i}\right)_{2 i+1}^{\uparrow \downarrow} \wedge\left(i \cdot B_{i}\right)_{2 i+1}^{\uparrow \downarrow}}{2}\right)
$$

The interaction is between the minimum limit kernel of the minimum structure group and life activities [16]. And $f_{\Sigma}\left(G_{2 i}^{\downarrow \uparrow}\right), f_{\Sigma}\left(E_{2 i}^{\downarrow \uparrow}\right)$ Continuous extension and combination [17] are the basis and condition for our life metabolism and obtaining regenerated cells (proteins). The significance of minimum limit kernel for protein repair and complex index there are numerous homomorphic limit kernel problems [18].

\section{The Relationship Between the Movement of Protein Particles and Cancer Tissue}

\subsection{Embedded Trigonometric Series (Function) Expansion Shows the Stability of Human Tissue}

$$
\operatorname{Cos}^{n-1}\left(\varphi_{1} \leftrightarrow P_{1}\right) \cdot \operatorname{Cos}^{n-2}\left(\varphi_{2} \leftrightarrow P_{2}\right) \cdot \operatorname{Cos}^{n-3}\left(\varphi_{3} \leftrightarrow P_{3}\right) \cdot \ldots \cdot \operatorname{Cos}^{1}\left(\varphi_{n} \leftrightarrow P_{n}\right)
$$

is the expansion of trigonometric series (function). When the cancer tissue appears

$$
\prod_{i=1}^{n} \operatorname{Cos}^{i-1}\left(\overline{\uparrow \downarrow}_{j=1}^{m} \varphi_{j} \leftrightarrow P_{j}\right)
$$

the balance is broken, and the active degree of protein particles is accelerated rapidly.

$$
\left|\lim _{\eta \rightarrow \infty}\left(\operatorname{Cos}\left(\frac{\pi}{2}-\frac{\pi}{4 \eta}+2 k \pi\right)\right)\right|
$$

this function shows that the activity of cancer cells is very high, the movement of forming protein particles is accelerated rapidly, and the metabolism of human body is accelerated. Because of

$$
\left|\lim _{\eta \rightarrow \infty}\left(\operatorname{Cos}\left(\frac{\pi}{2}-\frac{\pi}{4 \eta}+2 k \pi\right)\right)\right|
$$

activity, the balance of protein movement is broken. At this time, the cancer cells replicate in large quantities.

When the cancerous tissue is removed, the movement of protein particles. That is, the activity of cancer cells and the minimal limit nucleus of $G_{2 i}^{\downarrow \uparrow}, E_{2 i}^{\downarrow \uparrow}$ were repaired after tumor resection. Under the high activity of

$$
\lim _{\eta \rightarrow \infty}\left(\operatorname{Cos}\left(\frac{\pi}{2}-\frac{\pi}{4 \eta}+2 k \pi\right)\right)
$$

the tumor grows rapidly.

The relationship between protein activity $A_{1}^{\mp}$ and minimum limit nucleus $B_{1}^{\mp}$ when cancer cells are active, the activity of corresponding protein increases at the same time; when minimum limit nucleus $B_{1}^{\mp}$ is active, after protein activity $A_{1}^{\bar{\mp}}$ rises rapidly, the minimum limit nucleus $B_{1}^{\bar{F}}$ accelerates the repair of protein at the same time, but the activity of protein to cancer cells does not reach the balance, resulting in further rapid growth of tumor; At the same time, these proteins fold to form cancer cells to grow crazily, producing a large number of impurities. When $A_{1}^{\bar{T}} \leftrightarrow B_{1}^{\bar{T}}$ produces a large number of impurities, it will have a great negative impact on the human body.

The spatial structure relationship between $\sum_{i=1}^{n}\left(\frac{e^{+}}{e^{-}}\right)^{i-}$ and the minimum limit kernel. When

$$
\eta=\frac{1}{2+8 k}, k=1,2, \ldots, \sum_{i=1}^{n}\left(\frac{1}{(2 \pi)^{-1} \cdot \psi\left(\delta_{1} \leftrightarrow \delta_{2}\right)}\right)_{\text {Min }},
$$

can appear protein repair and replication to form confusion, and the tissue becomes irregulartumor. For impurity

$$
\sum_{i=1}^{n}\left(\frac{1}{(2 \pi)^{-1} \cdot \psi\left(\delta_{1} \leftrightarrow \delta_{2}\right)}\right)_{\text {Min }}
$$

the period exists.

When $\eta=\frac{1}{2+8 k}, k=1,2, \ldots$, after the limit period after impurity generation. Cancer cells are no longer controlled by the $\prod_{i=1}^{n} \operatorname{Cos}^{i-1}\left(\overline{\uparrow \downarrow}_{j=1}^{m} \varphi_{j} \leftrightarrow P_{j}\right)$ function. Then

$$
\frac{2 \pi}{\psi\left(f_{\Sigma}^{-}\left(G_{2 i}^{\downarrow \uparrow}\right) \leftrightarrow f_{\Sigma}^{+}\left(E_{2 i}^{\downarrow \uparrow}\right)\right)}
$$

The impurity formed by $\sum_{i=1}^{n}\left(\frac{e^{+}}{e^{-}}\right)^{i-}$ will interfere with the double stranded DNA. When $\eta=\frac{1}{2+8 k}, k=1,2, \ldots$, base pairs on the DNA strand, and dislocation may also occur in the arrangement.

$$
\psi\left(\sum_{i=1}^{n}\left(\frac{e^{+}}{e^{-}}\right)^{i-}, \frac{1}{2+8 k}\right), k=1,2, \ldots
$$

Impurity relation function is a very important function of human health.

$$
\prod_{i=1}^{n} \operatorname{Cos}^{i-1}\left(\overline{\uparrow \downarrow}_{j=1}^{m} \varphi_{j} \leftrightarrow P_{j}\right)
$$

Only when the protein particles move in the normal range can the human body get healthy.

When $\prod_{i=1}^{n} \operatorname{Cos}^{i-1}\left(\overline{\uparrow \downarrow}_{j=1}^{m} \varphi_{j} \leftrightarrow P_{j}\right) \quad$ invalid, and then $\psi\left(\sum_{i=1}^{n}\left(\frac{e^{+}}{e^{-}}\right)^{i-}, \frac{1}{2+8 k}\right)$, the human body will produce a large number of impurities and cancer cells, which will eventually lead to tumors and cancer. When $\eta=\frac{1}{2+8 k}, k=$ $1,2, \ldots$, It produces a lot of impurities and forms the limit cycle 
of cancer cells.

\subsection{Growth Rate of Impurities and Cancer Cells}

After the impurities and cancer cells exceeded $100 \%$, the growth rate $\operatorname{was} \sum_{i=1}^{n}\left(\frac{e^{+}}{e^{-}}\right)^{i-}$ class.

$$
\sum_{i=1}^{n}\left(\frac{e^{+}}{e^{-}}\right)^{i-} \cdot \frac{1}{2 \pi} \cdot \psi\left(f_{\Sigma}^{-}\left(G_{2 i}^{\downarrow \uparrow}\right) \leftrightarrow f_{\Sigma}^{+}\left(E_{2 i}^{\downarrow \uparrow}\right)\right)>1
$$

Impurities and cancer cells reached $100 \%$, with the lowest growth rate of $\left[\sum_{i=1}^{n}\left(\frac{e^{+}}{e^{-}}\right)^{i-}\right]_{\mathrm{Min}^{+}}$grade, and in a relatively stable state of high growth.

$$
\left[\sum_{i=1}^{n}\left(\frac{e^{+}}{e^{-}}\right)^{i-}\right]_{\text {Min }^{+}} \cdot \frac{1}{2 \pi} \cdot \psi\left(f_{\Sigma}^{-}\left(G_{2 i}^{\downarrow \uparrow}\right) \leftrightarrow f_{\Sigma}^{+}\left(E_{2 i}^{\downarrow \uparrow}\right)\right)=1
$$

When $\eta<\frac{1}{2+8 k}, k=1,2, \ldots$, Impurities and the activity of cancer cells decreased (due to the influence of treatment measures); due to the situation of formula (9), cancer is a mathematical boundary that is difficult to cross from the mathematical model; large sample measurement of cancer cure rate, cure rate $0^{+} \%$.

\subsection{Statistical Problems of Impurities and Cancer Cells Falling to Healthy Level}

$\eta_{\text {Min }}=\frac{1}{2+8 k}$ The equation for impurities and cancer cells to decline to a healthy level. $\operatorname{Cos}\left(-\frac{\pi}{2}+4 \pi+7 k \pi\right)=$ 0 , measure the coefficient term of cancer cure rate statistics.

$$
\begin{aligned}
& {\left[\sum_{i=1}^{n}\left(\frac{e^{+}}{e^{-}}\right)^{i-}\right]_{\text {Min }^{+}} \cdot \frac{1}{2 \pi} \cdot(,) \operatorname{and} \operatorname{Cos}\left(-\frac{\pi}{2}+4 \pi+7 k \pi\right)=0(11)} \\
& T_{i}=\frac{\pi}{4} \otimes P_{i} \cdot \prod_{j=1}^{m}\left(\frac{e^{-}}{e^{+}}\right)^{j-}, i, j=1,2, \ldots \text { and } T_{i} \text { life cycle, } P_{i} \text { treatment. }
\end{aligned}
$$

Image analysis of curve group of trigonometric function in $(\pi / 2, \pi)$ part.

$$
T_{i} \rightarrow \frac{\pi}{4} \otimes P_{i} \cdot \operatorname{Sin}\left(\sum_{j=1}^{m} \theta^{j-}\right), \theta^{j-} \in\left(\frac{\pi}{2}, \pi\right), j=1,2, \ldots
$$

The equivalence relation of equation (13) above has errors when the number of samples is insufficient. The derivative of $\prod_{j=1}^{m}\left(\frac{e^{-}}{e^{+}}\right)^{j-}$ can eliminate the statistical error of the finite root curve and establish the (13) differential equation to eliminate the error.

\subsection{Eliminate the Error by Derivative and Differentiation of the Core Equation}

$$
\psi\left(p_{i}, e^{ \pm}, \theta^{j^{-}}\right) d \psi=\left[\frac{\pi}{4} \otimes P_{i} \cdot \operatorname{Sin}\left(\sum_{j=1}^{m} \theta^{j-}\right)-\frac{\pi}{4} \otimes P_{i} \cdot \prod_{j=1}^{m}\left(\frac{e^{-}}{e^{+}}\right)^{j-}\right]^{\partial}
$$

$\prod_{j=1}^{m}\left(\frac{e^{-}}{e^{+}}\right)^{j-}, \operatorname{Sin}\left(\sum_{j=1}^{m} \theta^{j-}\right)$ Compensation phase difference of equivalence relation [20].

$$
\left.p_{i} \cdot \operatorname{Cos}\left(\sum_{j=1}^{m} \theta^{j^{-}}\right)+\left(p_{i}\right)^{\prime} \cdot \operatorname{Sin}\left(\sum_{j=1}^{m} \theta^{j-}\right)=\left(p_{i}\right)^{\prime} \cdot\left[\prod_{j=1}^{m}\left(\frac{e^{-}}{e^{+}}\right)^{j-}\right]+p_{i} \cdot\left[\sum_{j=1}^{m} j \cdot\left(\frac{e^{-}}{e^{+}}\right)^{\left[\frac{m(m+1)}{2}-1\right.}\right]^{-}\right]
$$

The relationship between cancer life cycle, treatment measures and impurities. 


$$
\left\{\begin{array}{l}
\frac{\pi}{4} \otimes P_{i} \cdot \operatorname{Sin}\left(\sum_{j=1}^{m} \theta^{j-}\right)=\frac{\pi}{4} \otimes P_{i} \cdot \prod_{j=1}^{m}\left(\frac{e^{-}}{e^{+}}\right)^{j-}-\left[p_{i} \cdot \operatorname{Cos}\left(\sum_{j=1}^{m} \theta^{j^{-}}\right)+\left(p_{i}\right)^{\prime} \cdot \operatorname{Sin}\left(\sum_{j=1}^{m} \theta^{j-}\right)\right] \\
\frac{\pi}{4} \otimes P_{i} \cdot \prod_{j=1}^{m}\left(\frac{e^{-}}{e^{+}}\right)^{j-}=\frac{\pi}{4} \otimes P_{i} \cdot \operatorname{Sin}\left(\sum_{j=1}^{m} \theta^{j-}\right)+\left[p_{i} \cdot \operatorname{Cos}\left(\sum_{j=1}^{m} \theta^{j^{-}}\right)+\left(p_{i}\right)^{\prime} \cdot \operatorname{Sin}\left(\sum_{j=1}^{m} \theta^{j-}\right)\right]
\end{array}\right.
$$

Expression equation of lymphocyte $\theta^{j 1^{-}}$and exfoliated cell $\theta^{j 2^{-}}$[21].

$$
\left\{\begin{array}{l}
\psi\left(p_{i}, \theta\right)_{1}=\frac{\pi}{4} \otimes P_{i} \cdot \operatorname{Sin}\left(\sum_{j=1}^{m} \theta^{j_{1}^{-}}\right)+\left[p_{i} \cdot \operatorname{Cos}\left(\sum_{j=1}^{m} \theta^{j_{1}^{-}}\right)+\left(p_{i}\right)^{\prime} \cdot \operatorname{Sin}\left(\sum_{j=1}^{m} \theta^{j_{1}^{-}}\right)\right] \\
\psi\left(p_{i}, \theta\right)_{2}=\frac{\pi}{4} \otimes P_{i} \cdot \operatorname{Sin}\left(\sum_{j=1}^{m} \theta^{j_{2}^{-}}\right)+\left[p_{i} \cdot \operatorname{Cos}\left(\sum_{j=1}^{m} \theta^{j_{2}^{-}}\right)+\left(p_{i}\right)^{\prime} \cdot \operatorname{Sin}\left(\sum_{j=1}^{m} \theta^{j_{2}^{-}}\right)\right]
\end{array}\right.
$$

The relationship between impurity $\left(\frac{e^{-}}{e^{+}}\right)^{j-}$ and comprehensive physiological index of human body [22].

$$
\frac{\pi}{4} \otimes P_{i} \cdot \prod_{j=1}^{m}\left(\frac{e^{-}}{e^{+}}\right)^{j-}=\frac{1}{J} \sum_{j=1}^{n} \psi\left(p_{i}, \theta\right)_{j}
$$

\section{Conclusion}

The connection in the complexity of human genes is formed by the continuity of set potential. Thus, we can find the complex pairing of genomes, in which the weak order and law also exist. The continuity and controllability of the whole pairing potential of gene chain [23], the discontinuity with DNA gene fragment, and the continuity of DNA forming chromosome skeleton to life body ensure the relative stability of species [24].

\section{References}

[1] Zhang Wenxiu, Qiu Guofang, Uncertain Decision Making Based on Rough Sets, Beijing China, tsinghua university press, 2005: 1-255.

[2] Zheng Weiwei, Complex Variable Function and Integral Transform, Northwest Industrial University Press, 2011: 1-396.

[3] Lou Senyue, Tang Xiaoyan, Nonlinear Mathematical Physics Method, Beijing China, Science Press, 2006: 1-365.

[4] Zhu RongRong, Differential incremental equilibrium geometry protein granule Space folding, genome expression and cell modification, Fudan University, Volume 1, 2015-04-1: 1-112.

[5] LABPHTEB M. A., TriaBAT B. B., Methods of Function of a Complex Variable Originally published in Russian under the title, 1956, 2006: 1-287.

[6] Numerical Treatment of Multi-Scale Problems Porceedings of the $13^{\text {th }}$ GAMM-Seminar, Kiel, January 24-26, 1997 Notes on Numerical Fluid Mechanics Volume 70 Edited By Wolf Gang Hack Busch and Gabriel Wittum.

[7] Zhu RongRong, Differential incremental equilibrium geometry - Effects of Cerebral Groove and Protein Granule Motion on Thinking Space and Mental Activity , 4-Dimensional Super-high-end Super-spherical Convex Spherical Fiber Cluster, Residual Product-like Cluster Petal Microfibers, Fudan University, Volume 3, 2015-04-18: 1-227.

[8] C. Rogers W. K. Schief, Bäcklund and Darboux Transformations: Geometry and Modern Applications in Solition
Theory, first published by Cambridge University, 2015: 1-292.

[9] Guchaohao, $\mathrm{Hu}$ Hesheng, Zhou Zixiang, Dar Boux Transformation in Solition Theory and Its Geometric Applications (The second edition), Shanghai science and technology Press, 1999, 2005: 1-271.

[10] W. Miller, Symmetry Group and Its Application, Beijing China, Science Press, 1981: 1-486.

[11] Gong Sheng, Harmonic Analysis on Typical Groups Monographs on pure mathematics and Applied Mathematics Number twelfth, Beijing China, Science Press, 1983: 1-314.

[12] Ren Fuyao, Complex Analytic Dynamic System, Shanghai China, Fudan University Press, 1996: 1-364.

[13] Chen Zhonghu, Lie group guidance, Higher Education Press, 1997: 1-334.

[14] Su Jingcun, Topology of Manifold, Wuhan China, wuhan university press, 2005: 1-708.

[15] Wu Chuanxi, Li Guanghan, Submanifold geometry, Beijing China, Science Press, 2002: 1-217.

[16] Ding Peizhu, Wang Yi, Group and its Express, Higher Education Press, 1999: 1-468.

[17] Zheng jianhua, Meromorphic Functional Dynamics System, Beijing China, tsinghua university press, 2006: 1-413.

[18] Xiao Gang, Fibrosis of Algebraic Surfaces, Shanghai China, Shanghai science and technology Press, 1992: 1-180.

[19] Jari Kaipio Erkki Somersalo, Statistical and Computational Inverse Problems With 102 Figures, Spinger.

[20] E. M. Chirka, Complex Analytic Sets Mathematics and Its Applications, Kluwer Academic Publishers Gerald Karp, Cell and Molecular Biology: Concepts and Experiments (3e), Higher Education Press, 2005: 1-792.

[21] Zhu RongRong, Differential incremental equilibrium geometry-protein granule Space folding, genome expression and cell modification General solution of nonlinear class of isolated wavelet -- effective nuclear trace information, Fudan University, Volume 2, 2015-04-11: 1-185.

[22] Qiu Chengtong, Sun Licha, Differential Geometry Monographs on pure mathematics and Applied Mathematics Number eighteenth, Beijing China, Science Press, 1988: 1-403.

[23] ShouTiande, Neurobiology, Higher Education Press, 2001, 2003: 1-470.

[24] Shou Tiande, Neurobiology (2e), Higher Education Press, 2001, 2006: 1-548. 